\title{
Oxaliplatin resistance is enhanced by saracatinib via upregulation Wnt-ABCG1 signaling in hepatocellular carcinoma
}

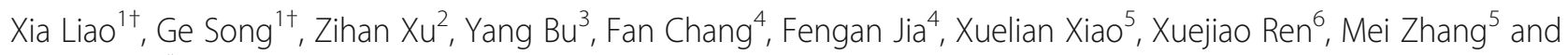
Qingan $\mathrm{Jia}^{5^{*}}$ (D)

\begin{abstract}
Background: Chemo-resistance in hepatocellular carcinoma (HCC) is a major problem, and acquired drug resistance prevents cancer therapies from achieving complete responses. Molecular targeting therapy presents an opportunity to impede tumor through combination or sequential therapy, while the accurate effect is vague.

Methods: The efficacy of combinations between oxaliplatin and anti-cancer molecular targeting drugs was screened. Strangely, the combined chemotherapy with oxaliplatin and saracatinib induced significantly antagonistic effects. Then the antitumor effects of combined treatment with saracatinib and oxaliplatin were confirmed in wide type HCC as well as in saracatinib- and oxaliplatin-resistant HCC. RNA sequencing was used to explore the resistance mechanism, and the roles of ATP-binding cassette transporter G1 (ABCG1) and Wnt signaling in oxaliplatin resistance were confirmed.

Results: Chemotherapy with oxaliplatin and saracatinib individually induced strong anti-HCC effects, while combined or sequential treatment of HCC cells with these two drugs exhibited reduced efficacy compared to treatment with the single drugs. And it was saracatinib treatment caused oxaliplatin resistance. RNA sequencing revealed 458 genes that were altered by treatment with saracatinib and oxaliplatin. Of these, the gene encoding ABCG1 and Wnt-associated genes were significantly upregulated. Upregulation of $A B C G 1$ and oxaliplatin resistance were associated with activation of Wnt signaling. Interference with $A B C G 1$ expression or inhibition of Wnt signaling resulted in reversal of the saracatinib-induced oxaliplatin resistance in HCC.

Conclusions: These studies demonstrated that combined or sequential chemotherapy with oxaliplatin and saracatinib reduced antitumor efficacy, and this antagonism was attributed to the activation of Wnt signaling and upregulation of $A B C G 1$ by saracatinib.
\end{abstract}

Keywords: Hepatocellular carcinoma, Oxaliplatin resistance, Saracatinib, Wht signaling, ABCG1

\section{Background}

In clinical practice, more than $70 \%$ of patients with $\mathrm{HCC}$ are diagnosed at an advanced stage and are treated with a non-radical surgical regimen, including transcatheter arterial chemoembolization (TACE) and systemic chemotherapy [1]. Oxaliplatin has commonly been used, although the efficacy of oxaliplatin for HCC is poor, due to the presence of both intrinsic and acquired resistance.

\footnotetext{
* Correspondence: qajia66@163.com

${ }^{+}$Xia Liao and Ge Song contributed equally to this work.

${ }^{5}$ Department of Hepatobiliary Surgery, First Affiliated Hospital of Xi'an

Jiaotong University, 277 West Yanta Road, Xi'an 710061, China

Full list of author information is available at the end of the article
}

Oxaliplatin resistance in HCC is a major medical problem, and methods for improvement of the response to this chemotherapeutic are urgently needed [2]. Molecular targeting therapy presents a therapeutic opportunity to impede tumor relapse and reverse drug resistance, while the accurate combined effect is not yet clear in HCC.

In this study, the efficacy of combinations between oxaliplatin and anti-cancer molecular targeting drugs was screened, and saracatinib treatment actually induced resistance to oxaliplatin treatment was proved. Then we evaluated the response of HCC to oxaliplatin and saracatinib in vitro and in vivo, and RNA sequencing revealed that the antagonistic relationship between saracatinib 
and oxaliplatin stemmed from activation of the Wnt signaling pathway, resulting in increased expression of the ATP-binding cassette transporter G1 ABCG1. Finally, we proved interference with $A B C G 1$ expression or inhibition of Wnt signaling resulted in reversal of the saracatinib-induced oxaliplatin resistance in HCC. These findings indicate that combination or sequential therapy with oxaliplatin and saracatinib have negative effects on $\mathrm{HCC}$ via upregulation Wnt-ABCG1 signaling.

\section{Methods}

\section{Cell lines and animals}

Human HCC cell lines MHCC97L, which has high metastatic potential (established at Fudan University, Shanghai, China; RRID: CVCL_4973), and Hep3B, which has low metastatic potential (American Type Culture Collection, Rockville, MD, USA; RRID: CVCL_0326), were obtained from the Liver Cancer Institute of Fudan University (Shanghai, China). All cells were maintained in Dulbecco's Modified Eagle's Medium (DMEM; GIBCO, Grand Island, NY, USA) and supplemented with $10 \%$ fetal bovine serum (FBS; GIBCO) at $37^{\circ} \mathrm{C}$ in a humidified incubator with $5 \% \mathrm{CO}_{2}$. Cells were routinely screened for the presence of mycoplasma (Mycoplasma Detection Kit, Roche Diagnostics, Indianapolis, IN, USA).

Male BALB/c nu/nu mice (aged 4-6 weeks and weighing approximately $20 \mathrm{~g}$ ) were obtained from the Chinese Academy of Science (SLRC, Shanghai, China) and raised in a controlled environment with $25^{\circ} \mathrm{C}$ under standard pathogen-free conditions and a natural light/dark cycle (morning 8:00; afternoon 8:00), and were provided with water and standard diet. Animal protocols were approved by the ethics committee on Experimental Animals of Xi'an Jiaotong University.

\section{Reagents and antibodies}

Oxaliplatin, and Src inhibitor saracatinib (AZD0530) were used for the construction of drug-resistant cell lines, and other anti-cancer molecular targeting drugs were purchased from ApexBio (Houston, TX, USA) and Selleck (Houston, TX, USA). Monoclonal antibodies to the following proteins were used in western blot: Ecadherin, vimentin, PCNA, FZD8, DKK1, AXIN2, WNT6, and $\beta$-catenin (purchased from Abcam, Cambridge, MA, USA) and p-LRP6, GSK-3ß, AXIN2, cyclin D1, SRC, OCT4, ABCG1, and BCL-2 (purchased from Proteintech, Chicago, IL, USA).

\section{In vitro drug sensitivity assay}

MHCC97L cells were seeded in 96-well plates at 2500 cells per well. Twelve hours after plating, cells were treated with anti-cancer molecular targeting drugs library (including 29 inhibitors in PI3K, MAPK signaling et al). After $72 \mathrm{~h}$ of incubation at $37^{\circ} \mathrm{C}$ in a $5 \% \mathrm{CO} 2$ humidified incubator, cell viability was analyzed using Cell Counting Kit 8 (CCK8; Dojindo, Gaithersburg, MD, USA). The drugs were stored and diluted according to the manufacturers' instructions.

\section{Generation of oxaliplatin- and saracatinib-resistant HCC cell lines}

MHCC97L and Hep3B cells were grown in $T_{25}$ flasks and treated with saracatinib $(2 \mu \mathrm{mol} / \mathrm{L}$ and $1 \mu \mathrm{mol} / \mathrm{L})$ followed by the addition of increasingly higher concentrations of saracatinib until the MHCC97L cells became stably resistant to $4 \mu \mathrm{mol} / \mathrm{L}$ saracatinib and the Hep3B cells became stably resistant to $2 \mu \mathrm{mol} / \mathrm{L}$ saracatinib. These resistant cells were re-named MHCC97L-Src and Hep3B-Src. Oxaliplatin-resistant HCC cell lines were generated as previously described [3]. MHCC97L cells that were stably resistant to $2 \mu \mathrm{mol} / \mathrm{L}$ oxaliplatin were re-named MHCC97L-Oxa, and Hep3B cells that were stably resistant to $1 \mu \mathrm{mol} / \mathrm{L}$ oxaliplatin were re-named Hep3B-Oxa.

\section{RNA interference}

The siRNA duplexes for $A B C G 1$ were chemically synthesized by Qiagen, Inc. (Valencia, CA, USA). The following ABCG1 siRNA sequences were constructed: $5^{\prime}$ CGTGGATGAGGTTGAGACA-3' (forward) and 5' GGTGGACAACAACTTCACA-3' (reverse). Chemically synthesized mock siRNA (fluorescein-labeled, nonsilencing) was also purchased from Qiagen, Inc. The human full-length cDNA of $A B C G 1$ were obtained from Genesent (shanghai China) and then cloned into the $\mathrm{pCDH}$ lentiviral expression vector (System Biosciences). Using the In-Fusion HD Cloning Kit (Takara), the amplified fragment was inserted into the plasmid pCDH (between XbaI and EcoRI sites). Flag-tagged $A B C G 1$ in $\mathrm{pCDH}$ vector was from Genesent (shanghai China).

\section{Cell viability assay}

Wild-type MHCC97L and Hep3B cells were grown in 96-well plates in medium containing $2 \mu \mathrm{mol} / \mathrm{L}$ oxaliplatin and increasing concentrations of saracatinib for 24, 48, 72, and $96 \mathrm{~h}$. Additionally, wild-type MHCC97L and Hep3B cells were grown in medium containing $2 \mu \mathrm{mol} / \mathrm{L}$ saracatinib and increasing concentrations of oxaliplatin for $24,48,72$, and $96 \mathrm{~h}$. Cell proliferation assays were performed with CCK8. Results were expressed as absorbance of each well at $450 \mathrm{~nm}$ (OD450).

\section{Animal model and treatment procedures}

MHCC97L $\left(5 \times 10^{6}\right)$ were implanted subcutaneously into the upper left flank region of mice to establish subcutaneous xenografts. The synergistic effects of the combination therapy of oxaliplatin $(10 \mathrm{mg} / \mathrm{kg})$ and saracatinib $(20 \mathrm{mg} / \mathrm{kg})$ were evaluated. Twenty nude mice bearing 
subcutaneous xenografts were randomly divided into the control, oxaliplatin, saracatinib, and oxaliplatin + sorafenib groups ( $n=5$ per group). Tumor weights were evaluated in 4 weeks after the treatments. Another eighteen nude mice bearing subcutaneous xenografts were randomly divided into the oxaliplatin, oxaliplatin + safacatinib, oxaliplatin + saracatinib + siABCG1 groups $(n=$ 6 per group). Small interfering RNA ABCG1 was used for local injection every 10 days. Tumor weights were also evaluated in 4 weeks after the treatments. Intraperitoneal injection of pentobarbital $(5 \mathrm{mg} / \mathrm{kg})$ combined with cervical spondylolisthesis was used for the sacrifice of mice after the study.

\section{Colony formation}

To investigate the effect of combination treatment with saracatinib and oxaliplatin on HCC cells, MHCC97L and Hep3B $\left(1 \times 10^{3}\right.$ cells/well $)$ were plated in 6-well plates and cultured with DMEM containing 5\% FBS with saracatinib $(2 \mu \mathrm{mol} / \mathrm{L})$ and/or oxaliplatin $(2 \mu \mathrm{mol} / \mathrm{L})$. Culture medium was replaced every $3 \mathrm{~d}$, and the colonies were fixed with ice-cold $4 \%$ paraformaldehyde after $14 \mathrm{~d}$. Cells were stained with Giemsa (Sigma, St. Louis, MO, USA) and photographed at $\times 5$ magnification.

\section{RNA sequencing and bioinformatics analysis}

RNA sequencing (Shanghai OE Biotech Co. Ltd., China) was used to compared MHCC97L with MHCC97L-Src and MHCC97L with MHCC97L-Oxa. Principal components analysis (PCA) and hierarchical clustering were performed using the $\mathrm{R}$ program. PCA was used to visualize differences between groups. Hierarchical cluster analysis was used to evaluate a set of dissimilarities, serving as a "complete" method for analyzing different genes based on the same gene ontology (GO). The GO seq $\mathrm{R}$ package was used to perform $\mathrm{GO}$ enrichment analysis of different gene clusters. KEGG enrichment analysis of different gene clusters were implemented using the cluster Profiler R package, and the cutoff for significance was set as $p=0.05$. Raw sequencing data is publicly available at NCBI (GEO accession number GSE129071).

\section{Cell cycle assays}

MHCC97L cells were starved in serum-free medium for $24 \mathrm{~h}$ and then grown in oxaliplatin $(2 \mu \mathrm{mol} / \mathrm{L})$ and/or saracatinib $(2 \mu \mathrm{mol} / \mathrm{L})$ for $48 \mathrm{~h}$. Cell cycle analyses and quantification of genomic DNA fragmentation were performed using the Cell Cycle Detection Kit (KeyGen, Nanjing, China) according to the manufacturer's protocol. Cell cycle distributions were analyzed by flow cytometry using a Becton Dickinson FACS Calibur (Franklin Lakes, NJ, USA).

\section{Statistical analysis}

A two-sided Student's $t$-test was performed to evaluate the statistical significance of differences in means. Experiments were performed at least three times, and $p<0.05$ was considered statistically significant. Statistical analyses were performed using SPSS 15.0 software for Windows (SPSS Inc. Chicago, IL, USA).

\section{Results}

\section{Combination chemotherapy with oxaliplatin and} saracatinib exhibited antagonistic effects

The efficacy of combinations between oxaliplatin and anti-cancer molecular targeting drugs was screened. Strangely, the combined chemotherapy with oxaliplatin and saracatinib induced significantly antagonistic effects (Fig. 1a). The HCC cell lines MHCC97L and Hep3B exhibited significant anti-tumor effects with lower proliferation rates than wild-type cells following treatment with oxaliplatin or saracatinib individually; however, combination treatment with the two drugs resulted in a higher proliferation rate, indicating impaired antitumor efficacy with combined treatment (Fig. 1b). To confirm this phenomenon, plate colony formation assays were performed. Compared to untreated cells, colony diameters were significantly decreased following treatment with oxaliplatin $\quad(257.51 \pm 55.60 \mu \mathrm{m}$ vs. $705.16 \pm 170.81 \mu \mathrm{m}$; $p=0.041)$ or saracatinib $(287.57 \pm 71.36 \mu \mathrm{m}$ vs. $705.16 \pm$ $170.81 \mu \mathrm{m} ; p=0.0025)$ individually. Combination therapy, on the other hand, resulted in larger colony diameters than oxaliplatin $(455.16 \pm 86.12 \mu \mathrm{m}$ vs. $257.51 \pm$ $55.60 \mu \mathrm{m} ; p=0.0086)$ or saracatinib $(455.16 \pm 86.1 \mu \mathrm{m}$ vs. $287.25 \pm 71.36 \mu \mathrm{m} ; p=0.0245)$ treatment alone (Fig. 1c). Next, we analyzed the cell cycle distributions to further evaluate the observed changes in cell proliferation. The percentages of cells in S phase decreased following treatment with either oxaliplatin $(22.321 \pm 0.67 \%$ vs. $29.48 \pm$ $1.06 \% ; p<0.0001)$ or saracatinib $(23.59 \pm 1.76 \%$ vs. $29.48 \pm$ $1.06 \% ; p=0.003)$. Combination therapy with oxaliplatin and saracatinib resulted in significantly increased percentages of cells in $\mathrm{S}$ phase compared to treatment with oxaliplatin $(26.55 \pm 0.39 \%$ vs. $22.321 \pm 0.67 \% ; p<0.0001)$ or saracatinib $(26.55 \pm 0.39 \%$ vs. $23.59 \pm 1.76 \% ; p=0.0026)$ individually (Fig. 1d). Furthermore, the tumor weight of the subcutaneous xenografts were larger in the combination treatment group than oxaliplatin $(3.06 \pm 0.16 \mathrm{~g}$ vs. $1.51 \pm 0.39 \mathrm{~g} ; p=0.0005)$ or saracatinib $(3.06 \pm 0.16 \mathrm{~g}$ vs. $1.97 \pm 0.32 \mu \mathrm{m} ; p=0.0008$ ) treatment alone (Fig. 1e).

We next revalidated the effects of the combined therapy on the response of HCC cells. MHCC97L (130.6 \pm $16.62 \mu \mathrm{mol} / \mathrm{L}$ vs. $20.85 \pm 4.86 \mu \mathrm{mol} / \mathrm{L} ; p=0.0063)$ and Hep3B $(28.67 \pm 5.59 \mu \mathrm{mol} / \mathrm{L}$ vs. $5.29 \pm 1.29 \mu \mathrm{mol} / \mathrm{L} ; p=$ 0.0247 ) cells exhibited significantly increased $\mathrm{IC}_{50}$ values in response to oxaliplatin when treated with saracatinib. Interestingly, however, MHCC97L $(0.79 \pm 0.11 \mu \mathrm{mol} / \mathrm{L}$ 


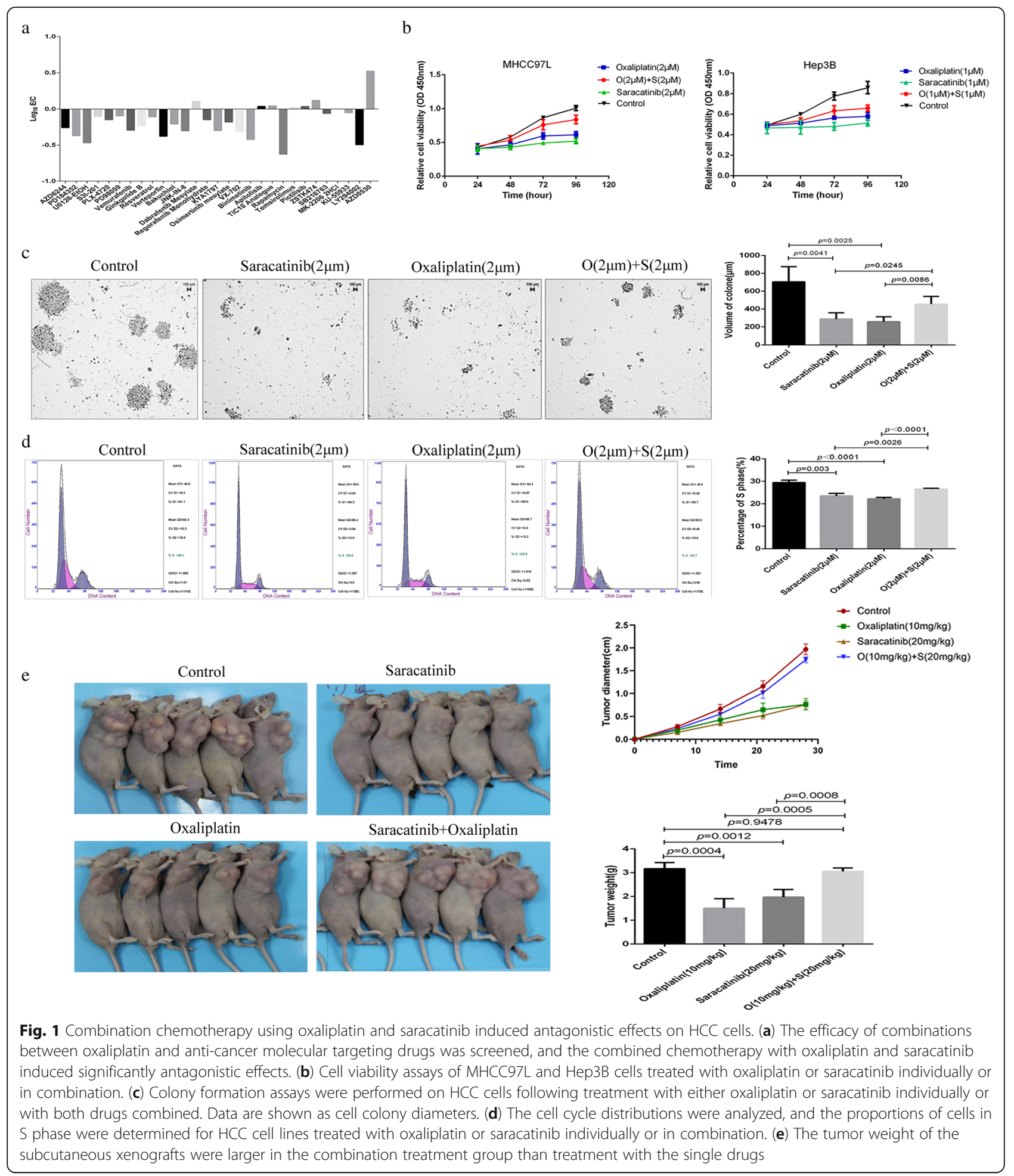

vs. $4.81 \pm 0.57 \mu \mathrm{mol} / \mathrm{L} ; p=0.0056)$ and Hep3B $(2.05 \pm$ $0.32 \mu \mathrm{mol} / \mathrm{L}$ vs. $2.62 \pm 0.47 \mu \mathrm{mol} / \mathrm{L} ; p=0.0631)$ cells exhibited reduced $\mathrm{IC}_{50}$ values in response to saracatinib when treated with oxaliplatin as well (Fig. 2a). These findings suggest that saracatinib treatment in combination with oxaliplatin increases oxaliplatin resistance in
HCC, and it was the treatment with saracatinib caused oxaliplatin resistance. Additionally, we investigated the effects of the two chemotherapy drugs on protein expression in the two HCC cell lines MHCC97L and Hep3B. Oxaliplatin treatment led to the downregulation of PCNA and the occurrence of EMT, which was 


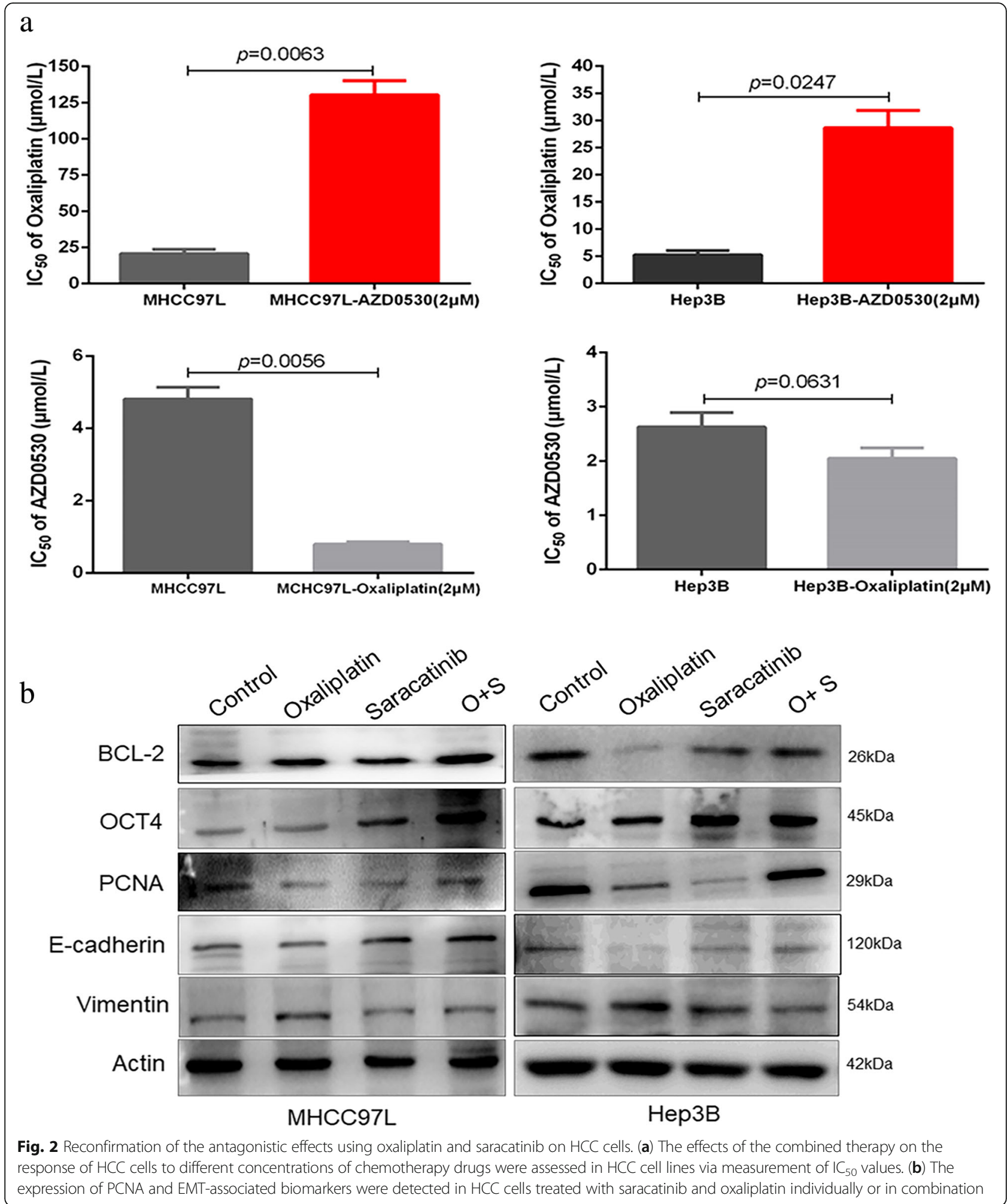

associated with the upregulation of vimentin and the downregulation of E-cadherin. Conversely, saracatinib not only downregulated PCNA expression but also reversed the EMT. MHCC97L and Hep3B treated with both oxaliplatin and saracatinib exhibited partial upregulation of PCNA and reversion of EMT (Fig. 2b). These findings suggest that saracatinib treatment in combination with oxaliplatin reduces the antitumor efficacy of 
these drugs on HCC cells, but reverses the negative effect of EMT induced by oxaliplatin.

\section{Sequential chemotherapy reduced the antitumor efficacy of oxaliplatin on saracatinib-resistant HCC}

In order to simulate the clinical sequential chemotherapy, HCC cell lines were treated continually with oxaliplatin to generate oxaliplatin-resistant cell lines (MHCC97L-Oxa and Hep3B-Oxa) that exhibited decreased intercellular adhesion and spindle-shaped cell morphology (Fig. 3A). Compared to wild-type HCC cells, MHCC97L-Oxa
$(66.67 \pm 9.01 \mu \mathrm{mol} / \mathrm{L}$ vs. $31.67 \pm 4.04 \mu \mathrm{mol} / \mathrm{L} ; p=0.0254 ;$ Fig. 3B, a) and Hep3B-Oxa $(19.21 \pm 2.69 \mu \mathrm{mol} / \mathrm{L}$ vs. $5.45 \pm$ $1.23 \mu \mathrm{mol} / \mathrm{L} ; p=0.0212$; Fig. 3B, c) exhibited increased oxaliplatin $\mathrm{IC}_{50}$ values. The oxaliplatin-resistant $\mathrm{HCC}$ cell lines were next treated with increasing concentrations of saracatinib, resulting in a decrease of $\mathrm{IC}_{50}$ values in MHCC97L-Oxa $(1.23 \pm 0.31 \mu \mathrm{mol} / \mathrm{L}$ vs. $4.30 \pm 0.97 \mu \mathrm{mol} / \mathrm{L}$; $p=0.0141$; Fig. $3 \mathrm{C}$, a) and Hep3B-Oxa $(1.14 \pm 0.11 \mu \mathrm{mol} / \mathrm{L}$ vs. $2.62 \pm 0.47 \mu \mathrm{mol} / \mathrm{L} ; p=0.0333$; Fig. $3 \mathrm{C}$, b) compared to the parental wild-type HCC cells, still exhibiting more sensitive to saracatinib.

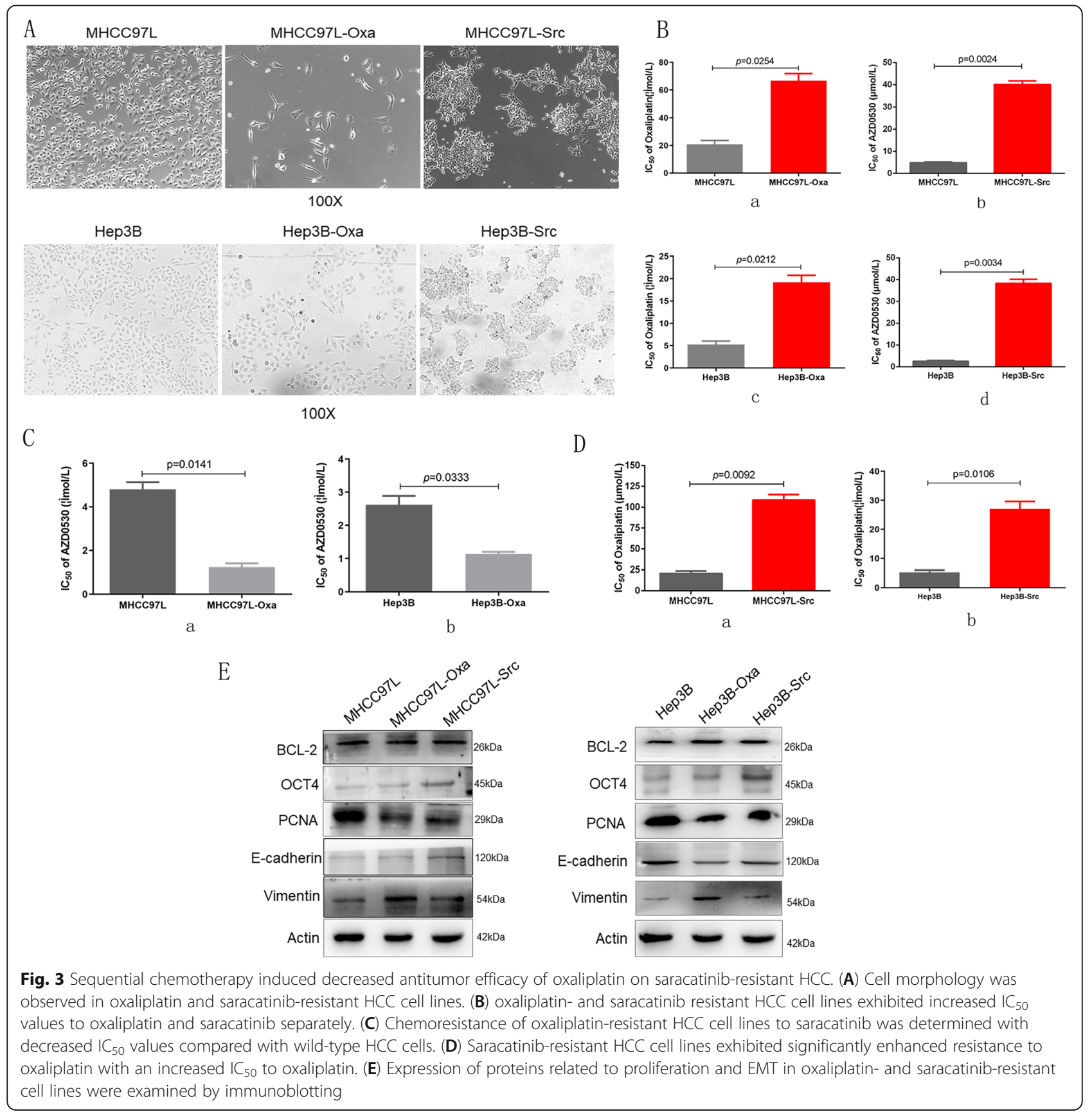


Saracatinib-resistant MHCC97L and Hep3B cells (MHCC97L-Src and Hep3B-Src) were generated similarly via continuous treatment with saracatinib, and these lines exhibited enhanced intercellular adhesion and appeared as agglomerated cell clumps (Fig. 3A). Compared to wildtype cells, MHCC97L-Src $(40.07 \pm 2.88 \mu \mathrm{mol} / \mathrm{L}$ vs. $4.81 \pm$ $0.57 \mu \mathrm{mol} / \mathrm{L} ; \quad p=0.0024 ;$ Fig. $3 \mathrm{~B}, \mathrm{~b})$ and Hep3B-Src $(38.36 \pm 3.17 \mu \mathrm{mol} / \mathrm{L}$ vs. $2.62 \pm 0.47 \mu \mathrm{mol} / \mathrm{L} ; p=0.009$; Fig. $3 \mathrm{~B}, \mathrm{~d})$ exhibited increased saracatinib $\mathrm{IC}_{50}$ values. The saracatinib-resistant $\mathrm{HCC}$ cell lines were then treated with oxaliplatin at increasing concentrations, yielding significantly enhanced resistance to oxaliplatin with increased $\mathrm{IC}_{50}$ in both MHCC97L-Src $(108.71 \pm 11.24 \mu \mathrm{mol} / \mathrm{L}$ vs. $20.85 \pm 4.86 \mu \mathrm{mol} / \mathrm{L} ; p=0.0092$; Fig. 3D, a) and Hep3B-Src $(27.01 \pm 4.59 \mu \mathrm{mol} / \mathrm{L} \quad$ vs. $5.29 \pm 1.29 \mu \mathrm{mol} / \mathrm{L} ; \quad p=0.0106$; Fig. 3D, b) compared to wild-type cells.

Additionally, we investigated protein expression in oxaliplatin- and saracatinib-resistant cell lines. MHCC97LOxa and Hep3B-Oxa exhibited downregulation of PCNA and E-cadherin and upregulation of vimentin and OCT4, while MHCC97L-Src and Hep3B-Src exhibited downregulation of PCNA, upregulation OCT4, and reversion of EMT compared to the parental cell lines (Fig. 3E). Together, these results indicate that sequential chemotherapy reduced the antitumor efficacy of oxaliplatin on saracatinib-resistant HCC.

\section{ABCG1 upregulation and Wnt signaling pathway activation are integral mechanisms involved in the antagonism between saracatinib and oxaliplatin in HCC}

The expression of 20,030 genes was compared between wild-type MHCC97L and MHCC97L-Src cells in three independent experiments (Fig. 4a). Gene expression profiles for 1172 genes exhibited differences $(p<0.05)$ between MHCC97L and MHCC97L-Src, implicating these genes in saracatinib resistance. Furthermore, analysis of these associated "resistance" genes revealed 526 upregulated and 645 downregulated genes. The expression of these 20,300 genes in wild-type MHCC97L and MHCC97L-Oxa was also compared (Fig. 4b). Expression profiles for 720 genes exhibited differences $(p<0.05)$ between MHCC97L and MHCC97L-Oxa, implicating these genes in oxaliplatin resistance. Of these, 455 were upregulated, and 265 were downregulated in the two drugresistant cell lines.

A total of 458 altered genes overlapped between the two drug-resistant cell lines, and all of these were closely related to cell division, growth, angiogenesis, adhesion, and metabolic processes (Fig. 4c). KEGG pathway analysis revealed that 20 of the altered genes were related to drug resistance: $A B C G 1, A T M, B B C 3, B I K, B I R C 3$, CDKN1A, DLL4, ERBB3, FGF2, FOS, GPER1, IL6, JAG1, MMP2, NRG2, PDGFRB, PIK3CA, SHC4, TOP2A, $T O P 2 B$, and VEGFA. Another 16 genes were related to
Wnt signaling: ROCK2, TCF7L1, WNT6, WNT5A, NKD2, FZD3, FZD8, DKK1, WNT5B, NFATC4, PLCB2, SERPINF1, AXIN2, NFATC1, PLCB4, and RAC2. Immunoblotting confirmed the upregulation of ABCG1 and Wnt-associated proteins, including FZD8, DKK1, Axin2, and WNT6 (Fig. 4d, e, f and g). Immunohisochemotherapy verified that the expression of ABCG1 was significantly upregulated after the treatment with oxaliplatin $(16.25 \pm 4.03$ vs. $7.50 \pm 3.42 \mu \mathrm{mol} / \mathrm{L} ; p=0.0162)$ or saracatinib (20.50 \pm 4.51 vs. $7.50 \pm 3.42 \mu \mathrm{mol} / \mathrm{L} ; p=0.0037)$ in subcutaneous xenografts tissues. And the combination treatment exhibited higher expression of ABCG1 than oxaliplatin single use $(30.50 \pm 5.01$ vs. $16.25 \pm 4.03 ; p=$ 0.0044; Fig. 4h). Therefore, we speculate that ABCG1 upregulation and Wnt signaling pathway activation are integral mechanisms involved in the antagonism between saracatinib and oxaliplatin in HCC.

\section{Interference with $A B C G 1$ expression or inhibition of Wnt signaling resulted in reversal of the saracatinib-induced oxaliplatin resistance in $\mathrm{HCC}$}

Immunoblotting verified that the expression of ABCG1 was significantly downregulated by $\mathrm{Wnt} / \beta$-catenin signaling pathway inhibition with KYA1797K in wild-type HCC cell lines, MHCC97L-Src, and Hep3B-Src (Fig. 5A). Following ABCG1 downregulation and, the key cell membrane receptors for Wnt signaling LRP6 and pLRP6 were not significantly altered; however, $\beta$-catenin was slightly downregulated, and the expression of PCNA was significantly decreased. ABCG1 restoration could reverse this alteration in protein levels (Fig. 5B).

Next, we confirmed the role of ABCG1 and Wnt signaling in oxaliplatin resistance. ABCG1 was silenced using specific siRNA in MHCC97L cells, resulting in a decreased $\mathrm{IC}_{50}$ to oxaliplatin compared to mock-treated MHCC97L cells (MHCC97L-Mock; $8.41 \pm 2.09 \mu \mathrm{mol} / \mathrm{L}$ vs. $25.59 \pm 5.82 \mu \mathrm{mol} / \mathrm{L} ; p=0.0085$; Fig. $5 \mathrm{C}$, a). Following silencing of ABCG1 using siRNA in saracatinib-resistant MHCC97L (MHCC97L-Src-ABCG1-Sh1), we observed decreased resistance to oxaliplatin $(40.43 \pm 8.12 \mu \mathrm{mol} / \mathrm{L}$ vs. $103.71 \pm 8.74 \mu \mathrm{mol} / \mathrm{L} ; p=0.0008$; Fig. $5 \mathrm{C}$, b) compared to mock-treated cells. Furthermore, silencing of ABCG1 combined with saracatinib in MHCC97L, there was no significantly increased resistance to oxaliplatin $(11.84 \pm 2.11$ vs. $9.73 \pm 1.26 \mu \mathrm{mol} / \mathrm{L} ; p=0.0874$; Fig. $5 \mathrm{C}, \mathrm{c})$. Additionally, HCC cells treated with the Wnt signaling inhibitor KYA1797K exhibited decreased resistance to oxaliplatin with reduced $\mathrm{IC}_{50}$ values $(11.07 \pm 2.02 \mu \mathrm{mol} / \mathrm{L}$ vs. $31.67 \pm 4.04 \mu \mathrm{mol} / \mathrm{L} ; p=0.0082$; Fig. $5 \mathrm{D}$, a). Treatment of MHCC97L-Src cells with KYA1797K resulted in decreased resistance to oxaliplatin $(40.83 \pm 8.12 \mu \mathrm{mol} / \mathrm{L}$ vs. $108.71 \pm 11.24 \mu \mathrm{mol} / \mathrm{L} ; p=0.0008$; Fig. 5D, b). KYA1797K combined with saracatinib in MHCC97L, there was no 


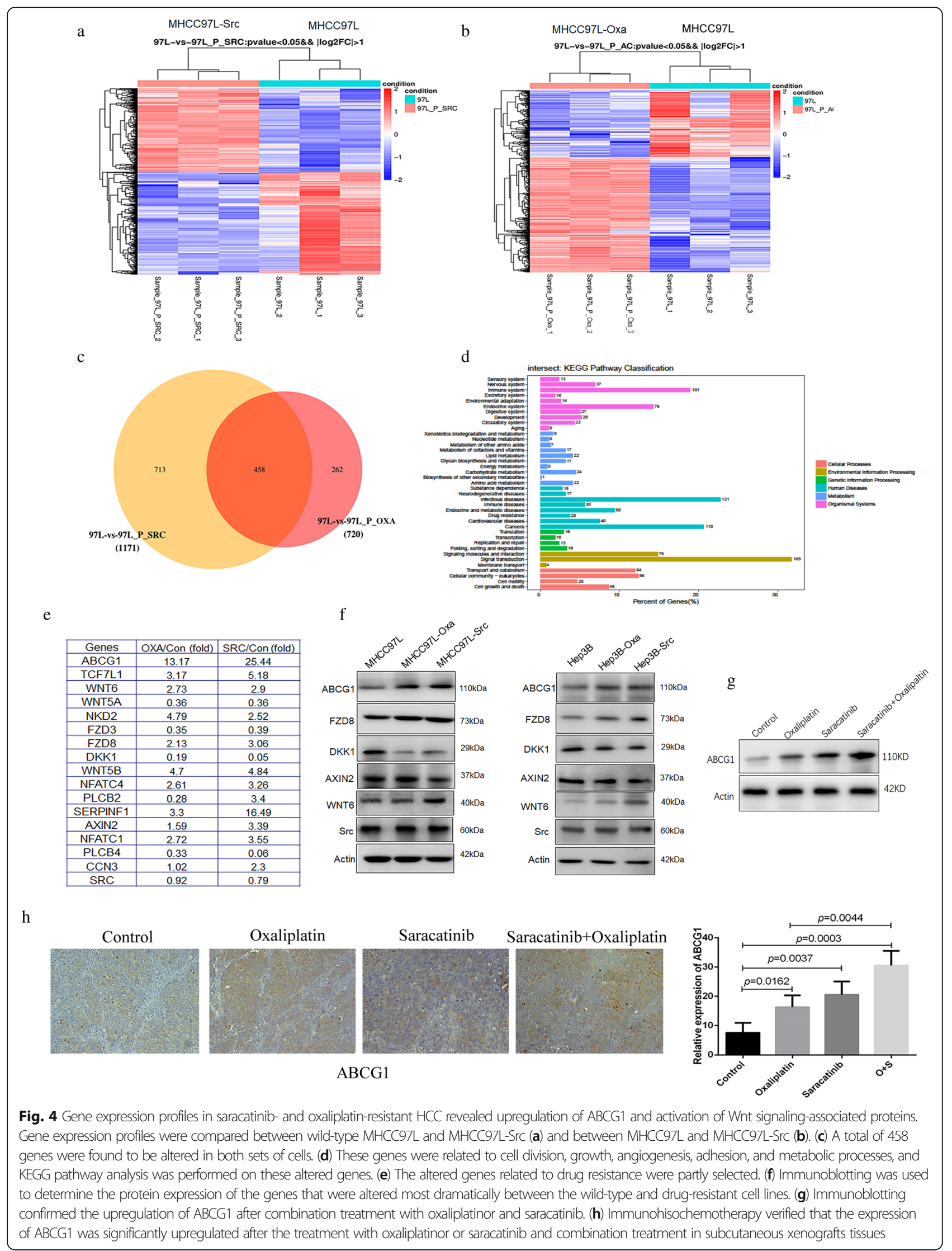




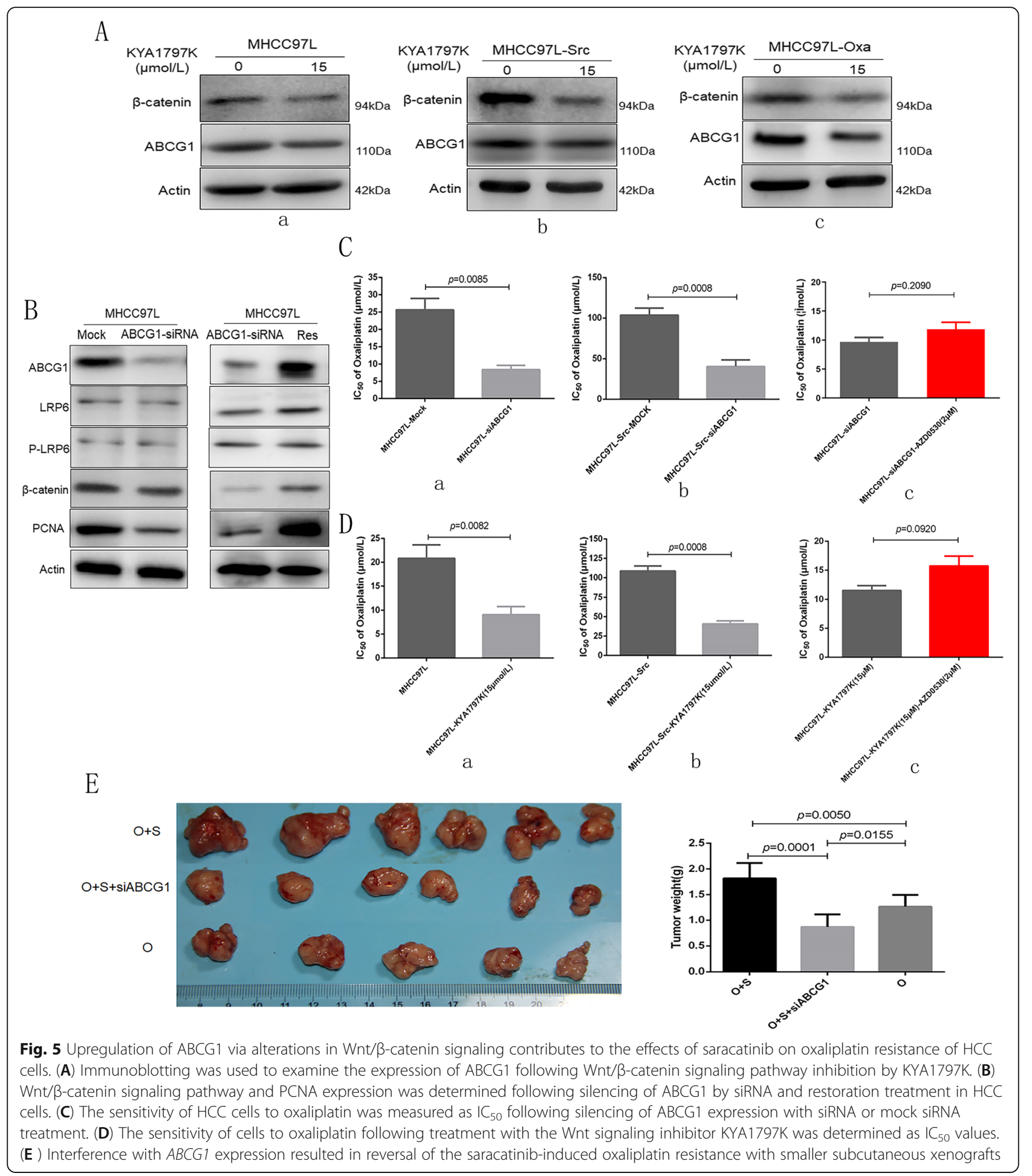

significantly increased resistance to oxaliplatin (11.51 \pm 1.44 vs. $15.73 \pm 2.95 \mu \mathrm{mol} / \mathrm{L} ; p=0.0920$; Fig. $5 \mathrm{D}, \mathrm{c}$ ).

Furthermore, it was confirmed once again that the tumor weight of the subcutaneous xenografts was larger in combination treatment group using oxaliplatin and saracatinib than oxaliplatin single use $(1.82 \pm 0.30 \mathrm{~g}$ vs.
$1.26 \pm 0.23$ g; $p=0.0050$; Fig. $5 \mathrm{E})$. And the subcutaneous xenografts was smaller in the combination treatment group using oxaliplatin, saracatinib, and ABCG1 siRNA local injection $(0.87 \pm 0.24 \mathrm{~g}$ vs. $1.82 \pm 0.30 \mathrm{~g} ; p=0.0001$; Fig. $5 \mathrm{E}$ ) than combination group only using oxaliplatin and saracatinib. Together, these findings suggest that 
ABCG1 and Wnt signaling contribute to oxaliplatin resistance in saracatinib-treated HCC cells. And interference with $A B C G 1$ expression or inhibition of Wnt signaling resulted in reversal of the saracatinib-induced oxaliplatin resistance in $\mathrm{HCC}$.

\section{Discussion}

Liver cancer, most commonly seen as hepatocellular carcinoma (HCC), has high prevalence and incidence rates in China, which accounts for more than $50 \%$ of the total number of liver cancer cases and deaths in the world [4]. One of the chemotherapeutic drugs for patients with advanced HCC is oxaliplatin, which initiates apoptosis by inhibiting the replication and transcription of DNA in HCC cells [5]. However, the efficacy of oxaliplatin on HCC is poor, exhibiting intrinsic and acquired resistance. Therefore, methods to enhance oxaliplatin treatment responses are urgently needed. In this study, the efficacy of combinations between oxaliplatin and anti-cancer molecular targeting drugs was screened, and saracatinib treatment actually induced resistance to oxaliplatin treatment was proved.

In many solid tumor cells, including HCC, Src expression level or activity is increased [6-8], promoting metastasis $[9,10]$. Activation of the tyrosine kinase Src is responsible for tumor progression promoted by insulinlike growth factor 1 receptor (IGF-1R) [11, 12]. Previously, we demonstrated that high expression of IGF1 was closely associated with the maintenance of stemness in oxaliplatin-resistant HCC cells and that IGF1-IGF1R signaling blockade effectively increased oxaliplatin sensitivity [3]. Tyrosine kinase Src was discovered more than 30 years ago as a kinase that is involved in the crosstalk between many signaling pathways, including the integrin/FAK, Ras/Raf/MEK, PI3K/AKT, and IGF1/IGF1R pathways, and Src activation promotes cell proliferation, adhesion, invasion, migration, metastasis, and tumorigenesis [13]. Recently, Liu et al. [14] reported that increased expression of Src potentiates ERK activation and reverses sorafenib resistance in HCC. Thus, inhibition of Src may provide a new strategy for drug combination studies for HCC treatment [15]. Based on these findings, we expect a synergistic relationship between oxaliplatin and Src kinase inhibition. Saracatinib (AZD0530) is a potent, orally administered small molecule that inhibits Src by blocking the ATP binding site of the kinase [16]. However, the combined chemotherapy with oxaliplatin and saracatinib induced significantly antagonistic effects. Recent research proved saracatinib failed to demonstrate monotherapeutic efficacy, with undesirable stem cellpromoting functions in patients with head and neck squamous cell carcinoma [17]. In the present study, we proved the combined treatment of HCC with oxaliplatin and saracatinib impaired the efficacy of either drug individually. And it was mainly saracatinib treatment increased oxaliplatin resistance in HCC. We also tested the effects of the sequential treatment of HCC with oxaliplatin and saracatinib, and sequential chemotherapy also reduced the antitumor efficacy of oxaliplatin on saracatinib-resistant HCC.

Based on previous and our current findings, oxaliplatinresistant HCC cells exhibited decreased intercellular adhesion and spindle-shaped cell morphology that are characteristic of EMT [18], while saracatinib-resistant HCC cells exhibited enhanced intercellular adhesion and cell clumping [19]. Immunoblotting further confirmed that oxaliplatin treatment led to the occurrence of EMT as vimentin was upregulated and E-cadherin was downregulated. Saracatinib significantly inhibited the expression of PCNA and reversed the EMT. Gene expression analysis revealed 458 genes that were altered in both saracatinib- and oxaliplatin-resistant $\mathrm{HCC}$, and these genes were related to processes of cell division, growth, angiogenesis, adhesion, and metabolism. Based on KEGG pathway classification, 20 of these genes were related to drug resistance, while 16 were related to Wnt signaling activation. Furthermore, immunoblotting revealed that ABCG1 and the Wnt/B-catenin signaling pathway were both upregulated in HCC cell lines in the presence of continuous treatment with saracatinib or oxaliplatin.

ABCG1 is a cholesterol lipid efflux pump that plays a well-known role in tumor growth, conferring chemoresistance to various malignant tumors [20]. Several $A B C$ transporters, including ABCG1, are associated with multidrug resistance (MDR), which is a major obstacle to the effective clinical treatment of cancer [21]. In the present study, ABCG1 is a downstream protein in Wnt/ $\beta$-catenin signaling and can be significantly downregulated by $\mathrm{Wnt} / \beta$-catenin signaling pathway. Interference with the expression of ABCG1 or inhibition of Wnt/ $\beta$ catenin results in decreased oxaliplatin resistance, supporting a role for these proteins in the acquired drug resistance of HCC. Understanding the molecular pathogenesis of HCC chemoresistance is key to improving patients' prognosis.

\section{Limitation}

In the present study, we demonstrated that combined or sequential chemotherapy with oxaliplatin and saracatinib induced antagonistic effects, while our study was only limited to liver cancer, and lack of deeper mechanistic knowledge of the actions of saracatinib. Therefore, several fundamental questions remain to be answered concerning the combined or sequential chemotherapy with the two drugs in the further study. (1) Whether antagonisms are limited to a few specific cancer species? (2) Deeper mechanisms involved in antagonistic effects of the two drugs are also needed be explored? (3) Can 
saracatinib be used in other diseases is also need to be answered? Still, our results do provide some important clues that may help guide drug selection and therapeutic strategy used in clinical treatments of cancer.

\section{Conclusions}

From our experimental results and our review of the literature, we propose the following conclusions. (1) The combined and sequential chemotherapy with oxaliplatin and saracatinib induces significantly antagonistic effects. (2) ABCG1 upregulation and Wnt signaling pathway activation are integral mechanisms involved in the antagonism between saracatinib and oxaliplatin in HCC. (3) Interference with ABCG1 expression or inhibition of Wnt signaling resulted in reversal of the saracatinibinduced oxaliplatin resistance in HCC.

\section{Abbreviations}

CCK8: Cell Counting Kit 8; DMEM: Dulbecco's Modified Eagle's Medium; FBS: Fetal bovine serum; GO: Gene ontology; HCC: Hepatocellular carcinoma; IGF-1R: Insulin-like growth factor 1 receptor; MDR: Multidrug resistance; PCA: Principal components analysis; TACE: Transcatheter arterial chemoembolization

\section{Acknowledgements}

Not applicable.

\section{Authors' contributions}

$X L, G S, Z H X, Y B, F C, F A J, X L X, X J R, M Z$ and QAJ contributed to the study design, analysis, and interpretation of data. QAJ conceived the study, $X L, G S$, ZHX and YB performed the majority of the experiments. FC, FAJ and XLX participated in statistical analysis. XJR and MZ participated in the establishment of the mice model. QAJ drafted and prepared the manuscript. All authors approved the final manuscript.

\section{Funding}

This research project was mainly supported by the National Natural Science Foundation of China (81502694). This research project was also partly supported by the Fundamental Research Funds for the Central Universities (1191329835), Postdoctoral Science Foundation of China (2015 M570330), and Key projects of Ningxia Natural Science Foundation (NZ15130). The funders had no role in the study design, data collection and interpretation, or decision to submit the work for publication.

\section{Availability of data and materials}

All data generated or analyzed during this study are included in this published article. The datasets used and/or analyzed and materials developed during the current study are available from the corresponding author by reasonable request.

\section{Ethics approval and consent to participate}

None of the cell lines used in the present study required ethics approval for their use. Ethical approval was obtained from the Research Ethics Committee of Xi'an Jiaotong University. (Permit Number:2016-439).

\section{Consent for publication}

Not applicable.

\section{Competing interests}

The authors declare that they have no competing interests.

\section{Author details}

'Department of Nutrition, First Affiliated Hospital of Xi'an Jiaotong University, Xi'an 710061, China. ${ }^{2}$ Department of Burns and Plastic Surgery, Shaanxi Provincial People's Hospital, Xi'an 710068, China. ${ }^{3}$ Department of Hepatobiliary Surgery, General Hospital, Ningxia Medical University, Yinchuan
750001, China. ${ }^{4}$ Metabolite Research Center, Shaanxi Institute of Microbiology, Xi'an 710043, China. ${ }^{5}$ Department of Hepatobiliary Surgery, First Affiliated Hospital of Xi'an Jiaotong University, 277 West Yanta Road, Xi'an 710061, China. ${ }^{6}$ Medical College of Yan'an University, Yan'an 716000, China.

Received: 30 May 2019 Accepted: 18 December 2019

Published online: 13 January 2020

\section{References}

1. Tsurusaki M, Murakami T. Surgical and Locoregional therapy of HCC: TACE. Liver Cancer. 2015;4(3):165-75.

2. Zhang $Y$, Huang $L$, Shi $H$, Chen $H$, Tao J, Shen $R$, Wang T. Ursolic acid enhances the therapeutic effects of oxaliplatin in colorectal cancer by inhibition of drug resistance. Cancer Sci. 2018;109(1):94-102.

3. Bu Y, Jia QA, Ren ZG, Zhang JB, Jiang XM, Liang $L$, Xue TC, Zhang QB, Wang $\mathrm{YH}$, Zhang $\mathrm{L}$, et al. Maintenance of Stemness in Oxaliplatin-Resistant Hepatocellular Carcinoma Is Associated with Increased Autocrine of IGF1. PLoS One. 2014;9(3):e89686.

4. Torre LA, Bray F, Siegel RL, Ferlay J, Lortet-Tieulent J, Jemal A. Global cancer statistics, 2012. CA Cancer J Clin. 2015;65(2):87-108.

5. Qin S, Bai Y, Lim HY, Thongprasert S, Chao Y, Fan J, Yang TS, Bhudhisawasdi $\checkmark$, Kang WK, Zhou Y, et al. Randomized, multicenter, open-label study of oxaliplatin plus fluorouracil/leucovorin versus doxorubicin as palliative chemotherapy in patients with advanced hepatocellular carcinoma from Asia. J Clin Oncol. 2013;31(28):3501-8.

6. Sievers E, Trautmann M, Kindler D, Huss S, Gruenewald I, Dirksen U, Renner M, Mechtersheimer G, Pedeutour F, Aman P, et al. SRC inhibition represents a potential therapeutic strategy in liposarcoma. Int J Cancer. 2015;137(11): 2578-88.

7. Roseweir AK, Qayyum T, Lim Z, Hammond R, MacDonald Al, Fraser S, Oades GM, Aitchison M, Jones RJ, Edwards J. Nuclear expression of Lyn, a Src family kinase member, is associated with poor prognosis in renal cancer patients. BMC Cancer. 2016;16:229.

8. Leroy C, Shen Q, Strande V, Meyer R, McLaughlin ME, Lezan E, Bentires-Alj M, Voshol H, Bonenfant D, Alex Gaither L. CUB-domain-containing protein 1 overexpression in solid cancers promotes cancer cell growth by activating Src family kinases. Oncogene. 2015;34(44):5593-8.

9. Zhao R, Wu Y, Wang T, Zhang Y, Kong D, Zhang L, Li X, Wang G, Jin Y, Jin $X$, et al. Elevated Src expression associated with hepatocellular carcinoma metastasis in northern Chinese patients. Oncol Lett. 2015;10(5):3026-34.

10. Zhao S, Li H, Wang Q, Su C, Wang G, Song H, Zhao L, Luan Z, Su R. The role of $\mathrm{c}$-Src in the invasion and metastasis of hepatocellular carcinoma cells induced by association of cell surface GRP78 with activated alpha2M. BMC Cancer. 2015;15:389.

11. Van Kolen K, Gilany K, Moens L, Esmans EL, Slegers H. P2Y12 receptor signalling towards PKB proceeds through IGF-I receptor cross-talk and requires activation of Src, Pyk2 and Rap1. Cell Signal. 2006;18(8):1169-81.

12. Sekharam M, Nasir A, Kaiser HE, Coppola D. Insulin-like growth factor 1 receptor activates C-SRC and modifies transformation and motility of colon cancer in vitro. Anticancer Res. 2003;23(2B):1517-24.

13. Anguita E, Villalobo A. Ca (2+) signaling and Src-kinases-controlled cellular functions. Arch Biochem Biophys. 2018;650:59-74.

14. Liu Y, Zhang X, Yang B, Zhuang H, Guo H, Wei W, Li Y, Chen R, Zhang N. Demethylation-induced overexpression of Shc3 drives c-Raf-independent activation of MEKJERK in HCC. Cancer Res. 2018;78(9):2219-32.

15. Xu L, Tong X, Zhang S, Yin F, Li X, Wei H, Li C, Guo Y, Zhao J. ASPP2 suppresses stem cell-like characteristics and chemoresistance by inhibiting the Src/FAK/snail axis in hepatocellular carcinoma. Tumour Biol. 2016;37(10): 13669-77.

16. Lang L, Shay C, Xiong Y, Thakkar P, Chemmalakuzhy R, Wang X, Teng $Y$. Combating head and neck cancer metastases by targeting Src using multifunctional nanoparticle-based saracatinib. J Hematol Oncol. 2018; 11(1):85.

17. Hermida-Prado F, Villaronga MA, Granda-Diaz R, Del-Rio-Ibisate N, Santos L, Hermosilla MA, Oro P, Allonca E, Agorreta J, Garmendia I, et al. The SRC Inhibitor Dasatinib Induces Stem Cell-Like Properties in Head and Neck Cancer Cells that are Effectively Counteracted by the Mithralog EC-8042. J Clin Med. 2019;8(8). https://doi.org/10.3390/jcm8081157.

18. Tanaka S, Hosokawa M, Yonezawa T, Hayashi W, Ueda K, Iwakawa S. Induction of epithelial-mesenchymal transition and down-regulation of miR- 
200c and miR-141 in oxaliplatin-resistant colorectal cancer cells. Biol Pharm Bull. 2015;38(3):435-40.

19. Yamaguchi H, Takanashi M, Yoshida N, Ito Y, Kamata R, Fukami K, Yanagihara K, Sakai R. Saracatinib impairs the peritoneal dissemination of diffuse-type gastric carcinoma cells resistant to met and fibroblast growth factor receptor inhibitors. Cancer Sci. 2014;105(5):528-36.

20. Namba Y, Sogawa C, Okusha Y, Kawai H, Itagaki M, Ono K, Murakami J, Aoyama E, Ohyama K, Asaumi JI, et al. Depletion of lipid efflux pump ABCG1 triggers the intracellular accumulation of extracellular vesicles and reduces aggregation and tumorigenesis of metastatic Cancer cells. Front Oncol. 2018;8:376

21. Li K, Lai H. TanshinonellA enhances the chemosensitivity of breast cancer cells to doxorubicin through down-regulating the expression of MDRrelated ABC transporters. Biomed Pharmacother. 2017;96:371-7.

\section{Publisher's Note}

Springer Nature remains neutral with regard to jurisdictional claims in published maps and institutional affiliations.

Ready to submit your research? Choose BMC and benefit from:

- fast, convenient online submission

- thorough peer review by experienced researchers in your field

- rapid publication on acceptance

- support for research data, including large and complex data types

- gold Open Access which fosters wider collaboration and increased citations

- maximum visibility for your research: over $100 \mathrm{M}$ website views per year

At BMC, research is always in progress.

Learn more biomedcentral.com/submissions 